\title{
Kernos
}

Revue internationale et pluridisciplinaire de religion grecque antique

5 | 1992

Varia

\section{Dionysos chez Eschyle}

François Jouan

ERdition électronique

DOI : 10.4000/kernos.1049

ISSN : 2034-7871

\section{Éditeur}

Centre international d'étude de la religion grecque antique

\section{Édition imprimée}

Date de publication : 1 janvier 1992

ISSN : 0776-3824

\section{Référence électronique}

François Jouan, « Dionysos chez Eschyle », Kernos [En ligne], 5 | 1992, mis en ligne le 19 avril 2011, consulté le 01 mai 2019. URL : http://journals.openedition.org/kernos/1049 ; DOI : 10.4000/ kernos. 1049 
Kernos, 5 (1992), p. 71-86.

\section{DIONYSOS CHEZ ESCHYLE}

C'est dans le courant du VIe siècle que le xoanon de Dionysos fut apporté à Athènes des frontières de la Béotie et que son culte se développa dans une série de fêtes qui comptèrent vite parmi les plus importantes de la cité. Quant au dieu lui-même, Pisistrate aidant, il s'affirma comme le patron des plus nobles genres poétiques, dithyrambe, tragédie, comédie et drame satyrique. De ce fait, on pouvait s'attendre à ce que la légende de Dionysos figurât parmi les plus anciens sujets dramatiques. Le paradoxe est cependant que la seule tragédie dionysiaque conservée date de l'extrême fin du Ve siècle : les Bacchantes, pièce posthume d'Euripide, représentée à Athènes en 405 . Les légendes dionysiaques n'en ont pas moins été présentes dans le théâtre attique à date ancienne. Si l'existence d'un Penthée de Thespis est douteuse ${ }^{1}$, la première moitié du Ve siècle a connu une Lycurgie de Polyphrasmon, représentée en $467^{2}$, et deux tétralogies d'Eschyle. Pour Sophocle, on ne peut citer que des drames satyriques, mais son fils Iophon avait produit des Bacchantes, à une date inconnue ${ }^{3}$. En 415, Xénoclès, avec encore des Bacchantes, l'avait emporté sur Euripide, qui présentait sa trilogie troyenne ${ }^{4}$. Dix ans plus tard, la pièce d'Euripide semble marquer un renouveau d'intérêt des poètes pour les sujets dionysiaques, qui se manifeste à travers plusieurs drames dans les décennies suivantes 5 .

On a tant écrit sur les Bacchantes d'Euripide, un de ses chefs-d'œuvre et une de nos sources majeures pour la connaissance des cultes dionysiaques, qu'il m'a paru préférable de me tourner vers Eschyle, dont les drames dionysiaques ont du reste constitué pour le plus récent des deux poètes une source plus importante qu'on ne l'a dit. Il est vrai que, si les pièces sont nombreuses - deux tétralogies entières plus au moins une ou deux tragédies, sans compter le drame satyrique des Spectateurs de

1 Cf. B. SNELL, $\operatorname{Tr} G F, 1,1971,1$ T24 et fr. 1-2, probablement une falsification d'Héraclide du Pont (Drog. L., V, 92).

2 Didascalie des Sept contre Thèbes (M).

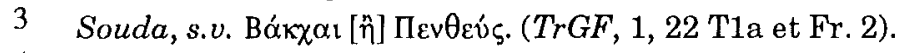

4 AEL., V.H., II, 8. La tétralogie de Xenocles comprenait Edipe, Lycaon, les Bacchantes et Athamas, drame satyrique.

5 Sémélé de Drogene ( $T r G F, 1,45$ F1; et de Carcinos II (170 F2-3)), Dionysos de Cheremon (71 F4-7), Bacchantes de Cleophon (77 T1). 
l'Isthme - les restes en sont fort minces. Par ailleurs, Dionysos est peu présent dans les pièces conservées.

Ce sont surtout les deux tétralogies qui nous retiendront. Dans l'état de nos connaissances, leur reconstitution pose des problèmes très difficiles, parfois même insolubles. On se trouve dès lors forcé de faire appel aux sources indirectes (récits de mythographes, représentations figurées) plus peut-être qu'il ne serait raisonnable. Quoi qu'il en soit, j'essaierai dans un premier temps de retracer, dans la mesure du possible, le sujet et l'action des deux tétralogies dionysiaques d'Eschyle. Je m'appuie, naturellement, sur les conclusions des nombreux travaux parus à ce jour ${ }^{6}$, sans exclure quelques hypothèses personnelles qui me paraissent rester dans le champ du vraisemblable. À partir de cettte analyse, et en me fondant directement sur les fragments conservés ${ }^{7}$, je tenterai de restituer l'image qu'Eschyle s'est formée et qu'il a voulu transmettre au public athénien du dieu des orgies, de sa légende et de son culte.

\section{La «Lycurgie»}

Chacune des deux tétralogies tournait autour du destin d'un adversaire de Dionysos, Lycurgue dans la Lycurgie, et Penthée dans l'autre groupe, qui n'avait pas reçu de nom générique. Il y a des raisons sérieuses de penser que dans chacune des tétralogies une tragédie (la seconde ou la troisième) s'écartait de la légende principale pour traiter un mythe apparenté, mais distinct : celui d'Orphée dans la première et celui des filles de Minyas dans la seconde. Certains critiques, il est vrai, ont rejeté cette hypothèse au nom de la cohérence interne de la trilogie, mais on répondra

6 Principales études : L. SECHAN, Etudes sur la Tragédie grecque, Paris, 1926; réimp. 1962, p. 73-79 (Lycurgie); 102-106 (Penthée); K. DEICHGRÄBER, in Nachr. Gött. Phil-Hist. Kl., 1, 3, 1938-1939, p. 231 sq. (Lycurgie); E.R. DoDDs, Euripides' Bacchae, Oxford, 1944, p. XxV-Xxx; H.J. METTE, Der Verlorene Aischylos, Berlin, 1963, p. 136-148; ID., in Lustrum, 9 (1964), p. 51-54; F. Ferrari, I Drammi perduti di Eschilo, Palerme, 1968, p. 367-388 (Lycurgie); A. LESKY, Die Tragische Dichtung der Hellenen, Göttingen, 1972², p. 153-154; T. GANZ, The Aeschylean Tetralogy : Attested and conjectured Groups, in AJPh, 101 (1980), p. 140-141; 153-158; M.L. West, Tragica VI, in BICS, 30 (1983), p. 63-72; R. AELION, Euripide héritier d'Eschyle, Paris, 1983, I, p. 249-257; B. Deforge, Eschyle poète cosmique, Paris, 1986, p. 141-151.

7 Cf. H.J. METTE, Die Fragmente der Tragödien des Aischylos, Berlin, 1959, p. 25-34 (fr. 69-100 : Lycurgie); p. 132-139 (fr. 354-372 : Dionysos-Dramen). Nous citerons les fragments d'après l'édition de S. RADT, TrGF, IV, Aischylos, Göttingen, 1983 : Edoniens : fr. 57-67; Bassarai, fr. 23-25; Néaniskoi, fr. 146149; Lycurgue, fr. 124-126. 
qu'Cdipe ne figurait pas dans tous les drames de l'Edipodie et Oreste dans tous ceux de l'Orestie.

La Lycurgie comprenait les Édoniens, les Bassarides, les Jeunes Gens (Néaniskoi) et Lycurgue, drame satyrique. La légende de Lycurgue était déjà connue d'Homère. L'Iliade rappelait sommairement que Lycurgue avait été aveuglé par Zeus pour avoir pourchassé les Nourrices de Dionysos et forcé le petit dieu à se jeter à la mer ${ }^{8}$. La version eschyléenne est très différente, et comporte en particulier une localisation précise : le héros est roi des Édoniens et vit en Thrace, dans la région du Pangée. On admet en général que cette tétralogie était postérieure à celle de Polyphrasmon, et qu'elle datait donc de la fin de la vie d'Eschyle, entre 466 et 459 . Or dans cette période, des forces athéniennes commandées par Cimon guerroyaient précisément contre les Édoniens, autour d'Eion et d'Ennéahodoi. Une tradition peu crédible, étant donné l'âge d'Eschyle, veut même que le poète ait participé à cette campagne. En tout cas, l'intérêt des spectateurs était attiré par cette région, qu'ils connaissaient et le poète avec eux - par les récits des combattants.

Les Édoniens sont la pièce la moins mal connue, en particulier du fait de l'existence de fragments d'un Lycurgue du poète latin Naevius, qui semble avoir suivi d'assez près le drame d'Eschyle ${ }^{9}$. Dans le prologue, un messager annonçait au roi thrace l'arrivée d'un personnage entouré d'un bruyant cortège de musiciens et de danseurs - Dionysos et son thiase. Le roi ordonnait de les arrêter et de les faire comparaître devant lui. Dans la parodos, le chœur, sans doute composé de sujets de Lycurgue, décrivait le cortège dionysiaque. Un garde rendait compte de l'arrestation des

8 Il., VI, 130-140 : «Lycurgue même, le puissant fils de Dryas, n'a pas vécu longtemps, du jour qu'il eut cherché querelle aux divinités célestes. N'avait-il pas un jour poursuivi les nourrices de Dionysos le Délirant sur le Nyséion sacré ? Toutes alors de jeter leurs thyrses à terre, sous l'aiguillon qui les poignait, de Lycurgue meurtrier, tandis qu'éperdu Dionysos plongeait dans le flot marin, où Thétis le reçut, épouvanté, dans ses bras; tant la peur l'avait pris au ton grondant de l'homme ! Mais contre celui-ci les dieux, qui vivent dans la joie, alors s'indignèrent; le fils de Cronos en fit un aveugle; et même ainsi il ne vécut pas longtemps : il était devenu un objet d'horreur pour tous les immortels» (trad. P. Mazon). Cf. H. Jeanmaire, Dionysos, Paris, 1951, p. 60-67.

9 Pour une resonstruction de la pièce, fondée en partie sur les fragments de Naevius, voir outre DeICHGräBER, op. cit., D.F. SUTTON, Aeschylos "Edonians", in Fons Perennis (Mél. V. D'Agostino), Turin, 1971, p. 387-411 (390-396). Sutton souligne à juste titre les rapprochements avec l'intrigue des Bacchantes. Il n'exclut pas que, comme Penthée, Lycurgue mourait avant la fin de la pièce. 
trublions et de leur docilité. Le roi interrogeait lui-même Dionysos, comme le Penthée des Bacchantes. Aussi mécontent des réponses de Dionysos que le prince thébain, il l'emprisonnait avec sa suite dans son palais, où il devait trouver, lui disait-il ironiquement "un logement gratuit» ${ }^{10}$. Mais la défaite du dieu n'était que provisoire. Bientôt les liens des captifs tombaient d'eux-mêmes, Dionysos apparaissait dans sa gloire sur le toit du palais, qui vacillait sur sa base et se nimbait de flammes : autre motif inclus dans nos Bacchantes ${ }^{11}$. On ne sait comment se terminait le drame. Je ne pense pas, contrairement à plusieurs interprètes, que la vengeance de Dionysos s'achevait dans cette pièce, déjà chargée de matière. Tout au plus le roi devait-il faire l'épreuve amère de son impuissance devant le dieu.

Les Bassarai ou Bassarides (les deux titres sont donnés parallèlement) tiraient leur nom de la bassara, terme thrace désignant le renard, et par extension une tunique longue en peau de renard, vêtement rituel ayant donné leur nom aux bacchantes thraces, adeptes d'un dieu plus tard appelé Dionysos Bassareus ${ }^{12}$. Le sujet en est discuté, car les rares fragments significatifs ne concernent que deux autres héros thraces, Orphée et Thamyris. L'hypothèse généralement admise est que l'histoire d'Orphée était présentée à Lycurgue comme un exemplum, destiné à le détourner de se dresser contre Dionysos. Il en aurait été de même pour celle de Thamyris, lui aussi châtié pour avoir imprudemment défié les Muses ${ }^{13}$. Mais Martin West a développé avec de solides arguments une thèse déjà proposée avant lui ${ }^{14}$ et qui fait d'Orphée le héros de ce drame. Il note qu'un récit des Catastérismes d'Eratosthène qui concerne la mort d'Orphée et se réfêre nommément à Eschyle a toutes les apparences d'une hypothésis tragique. Voici ce texte : "Orphée n'honorait pas Dionysos et honorait comme le plus grand des dieux Hélios, qu'il invoquait sous le nom d'Apollon. Se levant pendant la nuit, il montait à l'aurore sur la montagne appelée Pangée et attendait le lever du soleil pour être le premier à le voir (ou pour que ce fût la première chose qu'il vît). Dionysos s'en irrita et lui envoya les Bassarides, ainsi que le dit Eschyle, qui le

10 Fr. $63(\alpha \circ \delta \alpha \sigma \mu \circ)$.

11 Bacch., 586-599; 622-624; 633.

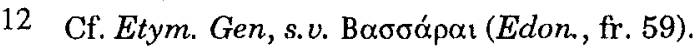

13 Parmi les partisans de cette solution, on citera entre autres F.G. Welcker, L. Séchan et R. Aélion.

14 Cf. M. West, op. cit., p. 64-70. C'était aussi l'avis d'Hermann, G. Murray, Th. Zielinski, K. Deichgräber, F. Ferrari, Casorran (BIEH, 2 (1968), p. 51-56) et D.F. Sutton. A. Lesky, H.J. Mette et A. Moreau (Eschyle, la violence et le Chaos, Paris, 1985, p. 218) préfèrent ne pas se prononcer. 
déchirèrent et dispersèrent ses membres. Les Muses, les ayant rassemblés, les enterrèrent au lieu qu'on appelle Leibethra» ${ }^{15}$. D'un examen attentif de certains commentaires anciens, West tira l'indication complémentaire qu'Orphée s'était écarté de Dionysos pour se tourner vers HéliosApollon à la suite de sa descente aux Enfers, où il avait eu des révélations sur le monde d'en-bas ${ }^{16}$.

L'action devait donc se passer sur Pangée. West a supposé un récit d'Orphée, peut-être juste de retour des Enfers, une parodos chantée par le chœur des Bassarides, et un stasimon évoquant l'histoire de Thamyris. D'autres scènes sont plus hypothétiques : une rencontre d'Orphée et de Lycurgue et un agôn entre Apollon et Dionysos. La mort d'Orphée déchiré par les Bassarides devait faire l'objet d'un récit de messager, ceci impliquant, comme dans nos Bacchantes, deux groupes distincts de Ménades, l'un en scène et l'autre hors de scène. Enfin, une Muse (Calliope ?) apparaissait ex machina, comme à la fin du Rhésos, tenant dans ses mains la tête d'Orphée.

Les Néaniskoi devaient tirer leur nom des jeunes Édoniens qui formaient le chœur, sans doute un groupe éphébique de sectateurs de Dionysos (plutôt que d'Apollon, comme le suggère West). À notre avis, c'est dans ce drame que s'accomplissait le châtiment de Lycurgue ${ }^{17}$. Un moment désarmé, le roi reprenait ses persécutions contre Dionysos et tentait d'interrompre par la force une fête dionysiaque nocturne ${ }^{18}$. Il y a sans doute plusieurs souvenirs de la pièce dans un stasimon de l'Antigone de Sophocle où le chœur énumère des précédents à l'emprisonnement de l'héroïne dans un caveau : "Il a dû plier sous le joug, le fils de Dryas aux trop promptes colères, le roi des Édoniens. Pour ses sarcasmes furieux, Dionysos l'enferma au fond d'un cachot. Il sent là tomber peu à peu l'élan féroce où jadis se déployait sa frénésie. Il a compris trop tard, au moment même où sa folie blessait le dieu de propos insultants. Il prétendait interrompre des femmes qu'inspirait le dieu, éteindre les torches que suit l'évohé; il provoquait les femmes amies des flûtes" ${ }^{19}$. Sophocle a cependant laissé de côté une partie du châtiment de Lycurgue, qui ne convenait

15 Ératosth., Astron., 24, p. 29 Ol.; cf. S. RADT, op. cit., p. 138.

16 Pour M. WEST, p. 68, Eschyle a pu avoir connaissance d'un poème orphique relatant la descente aux Enfers du héros et les révélations qu'il y avait obtenues.

17 Beaucoup de critiques placent l'action des Néaniskoi après le châtiment de Lycurgue (cf. L. SECHAN, op. cit., p. 77; A. LESKY, p. 154).

18 Allusion à une attaque nocturne de Lycurgue contre Dionysos et les Ménades (mais dans un autre contexte) chez DioDore, III, 66.

19

Ant., 955-965. 
pas à son sujet, et que l'on peut restituer d'après une série de vases attiques et italiotes illustrant la folie de Lycurgue sans aucun doute d'après une source tragique (en l'occurrence un récit de Messager) ${ }^{20}$. Au cours d'un sacrifice, Lycurgue saisi de folie furieuse (sur certains vases, Lyssa pointe sur lui un javelot), frappait de sa double hache son fils et sa femme et les tuait. On peut penser que les Jeunes Gens du chœur, sous la direction de Dionysos, s'emparaient ensuite de lui et l'enfermaient dans une grotte du mont Pangée ${ }^{21}$.

Reste le drame satyrique de Lycurgue, dont le souvenir est gardé par un vase attique et plusieurs vases italiotes représentant le héros au milieu des satyres. À quel moment de son histoire l'action se situait-elle? On a hésité entre deux possibilités : soit lorsque Dionysos se trouvait enfermé dans le palais du roi, entouré, comme le rappelle Apollodore, de Ménades et de satyres. Soit, plus vraisemblablement, à la fin de ses aventures, en supposant qu'au terme de son incarcération Lycurgue faisait amende honorable et se réconciliait avec Dionysos 22 . Un fragment présente le roi comme un buveur invétéré de bière thrace ${ }^{23}$. Sans doute le passage au vin était-il le signe concret de cette allégeance!

\section{La seconde tétralogie dionysiaque}

La reconstruction de ce second ensemble pose des problèmes plus difficiles encore, dans la mesure où sa composition même est conjecturale : cinq pièces, en effet, pourraient en faire partie : Sémélé ou les Porteuses d'Eau, Penthée, les Bacchantes, les Cardeuses de laine (Xantriai), et les Nourrices de Dionysos (Trophoi $)^{24}$. Il faut donc en éliminer une. Des diverses solutions proposées (erreur des sources, titres alternatifs ou

20 Après L. SEchan, p. 70-75, et A.D. TRENDALL - T.B.L. WeBster, Illustrations of Greek Drama, Londres, 1971, p. 49, 52 et pl. III, 1, 13-16, étudiés par D.F. SuTTON, in $R S C, 23$ (1975), p. 356-360, mais rattachés par le premier aux Bassarides et les deux autres aux Édoniens.

21 Chez Apollodore, III, 5, 1, une famine avait suivi ces meurtres. A la suite d'un oracle, les Édoniens avaient arrêté Lycurgue et l'avaient conduit sur le Pangée. Mais dans cette version, Dionysos l'avait fait déchiqueter par des chevaux.

22 Voir L. SECHAN, op, cit., p. 77-78; H.J. METTE, p. 140-141; R. AELION, p. 255. Une hydrie attique représente Lycurgue entouré de satyres (Fr. Brommer, Das Satyrspiele, Berlin, $1959^{2}$, p. 77, ${ }^{\circ} 97$ ).

23 Fr. 124

24 Sémélé, fr. 211-224 Radt (mais il attribue le fragment du P. Oxy. 2164 aux Xantriai); Penthée, fr. 183; Bacchantes, fr. 22; Xantriai, fr. 168-172b; Trophoi, fr. 246a-d. 
collectifs $)^{25}$, la plus simple parait être d'écarter les Bacchantes, dont il ne reste de toute manière que deux vers insignifiants.

Les Porteuses d'Eau, second titre de Sémélé, désignaient le chœur de servantes de la jeune princesse qui devaient apporter l'eau du bain destiné à l'enfant à naître, le petit Dionysos. Dans le drame, Sémélé était en butte au dénigrement et à la jalousie de ses sœurs, Agavé et Inô, qui refusaient de croire que le père de l'enfant était le roi des dieux, un thème qu'on retrouve au début des Bacchantes ${ }^{26}$. Un important papyrus ${ }^{27}$ nous a appris qu'Héra elle-même complotait contre la jeune femme. L'épouse de Zeus, déguisée en prêtresse-mendiante des nymphes de l'Inachos à Argos, se présentait à Thèbes et gagnait la sympathie des compagnes de Sémélé. Sur son conseil, les sœurs de la jeune femme la mettaient au défi de prouver ses dires en exigeant de son amant qu'il se présentât à elle dans toute sa gloire. Malgré les mises en garde d'une Nourrice dévouée, Sémélé se laissait prendre au piège et l'éclat fulgurant de Zeus la réduisait en cendres. Sans doute les Thébains étaient-ils persuadés que l'enfant avait péri avec sa mère. Je serais pour ma part tenté de rapporter au dénouement de Sémélé un vase attique du dernier quart du Ve siècle ${ }^{28}$ où l'on voit Hermès, devant la jeune femme qui s'effondre, s'emparer du bébé pour le confier aux nymphes de Nysa (dont l'une figure à l'arrière-plan), tandis que l'envoyée d'Héra, Iris, ne peut que constater qu'elle arrive trop tard pour achever la vengeance de l'épouse de Zeus.

Nouveau problème concernant le sujet des Xantriai. Ce titre a mené la critique sur une fausse piste, car on a pensé qu'il s'agissait d'Agavé et de ses bacchantes, qui "cardaient» le corps de Penthée comme un simple matelas de laine. Mais ce mot de "cardeuses» ne peut qualifier que la fonction des femme du chœur dès le début de la pièce ${ }^{29}$. On a alors pensé à une triade de victimes mémorables de Dionysos, les filles de Minyas, roi d'Orchomène de Béotie. Celles-ci se refusaient à célébrer les orgies dionysiaques avec les autres femmes de la ville, par amour de leur mari, nous disent les mythographes, et parce qu'elles se rangeaient sous les lois de

25 T. GANTZ, op. cit., p. 153-156, passe en revue les principales solutions proposées.

26 Bacch., 26-31.

27 Cf. H. LLOYD-JONEs, in Aeschylus, II, Loeb, p. 566-571. Attribué aux Xantriai, outre S. RADT (fr. 168), par E.R. Dodds, J. Roux et M. Lacroix, éditeurs des Bacchantes. Mais les arguments de K. LATTE, in Philologus, 97 (1948), p. 47 sq., me paraissent garder leur valeur.

28 Hydrie de l'Université de Californie : cf. R. MARTIN - H. Metzger, La Religion grecque, Paris, 1976, p. 134. Au registre supérieur siègent Zeus et Aphrodite.

29 Cf. T. GANZ, op. cit., p. 155. 
l'Athéna Erganè ${ }^{30}$. Elles restaient donc chez elles à travailler, c'est-à-dire à filer et à tisser la laine, ce qui explique la tâche de leurs servantes formant le chœur tragique. La colère de Dionysos déclenche dans la maison des prodiges terrifiants, elles sont prises d'une folie encore plus furieuse que celle des Ménades, puisqu'elles déchirent (et même dévorent, selon quelques sources), l'enfant d'une d'elles. Les Bacchantes d'Orchomène, Dionysos ou son prêtre à leur tête, poursuivent les trois folles et les tuent. Chez Ovide, elles sont changées en chauve-souris. La mort de Penthée sur le Cithéron était évoquée dans la pièce, mais sans doute à titre accessoire ${ }^{31}$.

La troisième tragédie aurait été un Penthée, dont l'action devait être proche de celle des Bacchantes d'Euripide. En tout cas, l'hypothésis de cette dernière pièce affirme, sous l'autorité d'Aristophane de Byzance, que "la mythopoiia est proche de celle du Penthée d'Eschyle», affirmation difficile à contrôler puisqu'il ne reste de ce Penthée qu'un seul vers ! Deux questions ont surtout préoccupé la critique : chez Eschyle, Agavé était-elle déjà associée aux ménades qui déchiraient Penthée ? Cela paraît au moins probable et pourrait même appartenir aux couches les plus anciennes de la légende ${ }^{32}$. Ensuite, on a cru trouver une référence au Penthée dans les vers fameux des Euménides où il est dit que Bromios "conduisit l'armée des Bacchantes et trama pour Penthée la mort d'un lièvre» 33 . On en a conclu que chez Eschyle une bataille rangée opposait les Ménades commandées par le dieu à la garde du roi de Thèbes. Il est vrai que ce motif apparaît comme un consilium irritum dans deux passages des Bacchantes $^{34}$. Ce n'est donc pas exclu, mais ni les représentations figurées de la mort de Penthée au Ve siècle, ni même l'expression employée dans les Euménides, n'imposent cette conclusion ${ }^{35}$.

Dans le drame satyrique des Nourrices de Dionysos, il semble que les satyres, représentés comme les vieux maris des nymphes de Nysa nourrices du dieu, bénéficiaient d'une cure de rajeunissement, grâce aux bons

30 Ael., V. H., III, 42; cf. aussi ANT. LiB., 10; PluT., Quaest. Gr., 38; Ov., Mét., IV, $1-42$ et $389-450$.

31 Pour certains critiques, le sujet de la pièce aurait été la mort de Penthée (à propos duquel le fr. $172 \mathrm{~b}$ précise qu'Eschyle l'avait ici située sur le Cithéron, et non sur le Parnasse). Mais quel aurait été alors le sujet de Penthée ?

32 Cf. É. COChE DE LA FERTÉ, Penthée et Dionysos, in R. BLOCH (éd.), Recherches sur les religions de l'Antiquité classique, Genève-Paris, 1980, p. 109.

33 Eum., 24-26.

34 Bacch., 50-52; 781-782.

35 Voir F. VIAN, Les origines de Thèbes, Paris, 1963, p. 161-162; R. AELION, op. cit., p. 251-252. 
soins de Médée ${ }^{36}$. On connaît les pouvoirs de la sorcière au chaudron, qui les avait mis au service d'Eson, le père de Jason, mais en avait refusé les effets à Pélias, après que les filles de celui-ci l'eurent pieusement découpé en morceaux. Un cratère d'Ancône, des environs de 450, semble s'inspirer de cette pièce ${ }^{37}$. L'analyse des documents iconographiques a conduit Erika Simon à penser que la cure de rajeunissement dépassait même son but "ayant accru leur force vitale», dit un des rares fragments de la pièce - en sorte que c'étaient de jeunes satyreaux à peine adolescents qui sortaient du chaudron magique... 38

On a encore imaginé que ces Trophoi auraient pu accompagner une autre trilogie dionysiaque, qui aurait été formée des Toxotides, de Sémélé et d'Athamas. Le thème commun de ces quatre pièces, d'après Timothy Gantz ${ }^{39}$ qui a fait cette suggestion il y a une dizaine d'années, serait la naissance ainsi que les toutes premières années du nouveau dieu. Les Toxotides traitaient de la mort d'Actéon, le fils d'Autonoé, sœur de Sémélé, dévoré par ses chiens pour avoir contemplé Artémis nue au bain. Mais, selon certaines sources, sa punition aurait été due aussi au fait qu'amoureux de sa tante Sémélé, il se serait trouvé en rivalité avec Zeus. Quant à Athamas, qui, avec sa femme Inô avait élevé le petit Dionysos après la mort de sa mère, il avait connu de grands malheurs causés par la rancune persistante d'Héra. L'association proposée par Gantz n'est pas illogique, mais comme il l'a reconnu lui-même, elle constitue une simple hypothèse de travail.

\section{Eschyle et Dionysos}

Quelles que soient les incertitudes et les lacunes, les analyses qui précèdent peuvent nous suggérer quelques réflexions sur les sentiments d'Eschyle à l'égard de Dionysos et du dionysisme.

D'abord, il est évident pour le poète, comme pour les Grecs de son temps, que Dionysos est un dieu jeune, un dieu dont la naissance ne remonte qu'aux temps, considérés comme historiques, où vivaient les héros, quelques générations à peine avant la guerre de Troie. Et il est né dans une ville grecque, peu éloignée d'Athènes, Thèbes de Béotie. Sa mère est une princesse thébaine, Sémélé, petite-fille du fondateur de Thèbes,

36 HyGIN, Fab., 182, 2; Ov., Mét., VII, 294-296.

37 Fr. BRoMmer, op. cit., p. 42; 44-45, n० 78.

38 Fr. 427 Mette; cf. E. SIMON, in SHAW, 1981, 5, p. 27, qui s'appuie sur la peinture d'un conge de Berlin, du P. D'Altamira (Pl. 16).

39 Op. cit., p. 156-158; B. DEFORGE, op. cit., p. 149-150, propose pour sa part un groupement Bacchantes, Athamas et Spectateurs de l'Isthme. 
Cadmos. Mais dans le drame qui portait son nom, le poète rappelait que la jeune femme, du moment où elle fut enceinte des œuvres de Zeus, fut "possédée par le dieu" et que les femmes qui lui touchaient le ventre devenaient à leur tour "possédées» 40 . Ainsi, dès le ventre de sa mère, Dionysos manifeste l'emprise qu'il ne cessera d'exercer sur le sexe féminin. Quant à la naissance du nouveau dieu, elle est particulièrement dramatique, avec une catastrophe prénatale qui demande des solutions inouies : la "couture de la cuisse" de Zeus et la double naissance, thème euripidéen, ou le transfert d'urgence sur le mont Nysa par Hermès, tradition eschyléenne qui semble la plus commune. De toute façon, le jeune dieu doit partir très loin de Thèbes, car le poète sait bien, comme son public, qu'il passe son enfance en Orient et que c'est après l'adolescence qu'il entame le voyage qui le ramènera en Grèce. Chacune des étapes de ce retour figure dans les pièces d'Eschyle : l'enfance à Nysa, la Lydie, dont il gardera un vêtement, la fine crocote; puis la Phrygie, la Thrace, avec la fourrure de renard qui annonce la nébride hellénique, la peau de faon ou la tunique de peau de bouc; ensuite la Béotie, avec l'Orchomène minyenne, Thèbes, et enfin Eleuthères, dernière étape avant son installation dans le sanctuaire athénien de Dionysos Eleuthéreus.

Ce qui frappe surtout chez Eschyle, c'est l'importance qu'il accorde à l'étape thrace de Dionysos, avec les quatre drames de la Lycurgie. Le poète s'est plu à souligner les ressemblances entre le dionysisme et les cultes de Cotys-Cotytô, mais aussi la particularité de la bassara des ménades thraces, l'importance religieuse du Pangée, la «montagne d'argent» ${ }^{41}$ qui fascinait les Athéniens. L'histoire d'Orphée s'appuyait en partie sur une réalité religieuse thrace, le culte du Soleil dans ces populations. Le passage de Dionysos chez Lycurgue préfigurait aussi les obstacles que le dieu allait trouver sur sa route, pour établir son culte en Grèce. Mais dès l'étape thrace, sa personnalité divine est dessinée, le climat religieux du dionysisme et ses rites sont fixés dans toute leur spécificité.

L'apparence physique de Dionysos surprenait Lycurgue, comme il surprendra Penthée. "D'où vient cet "homme-femmelette" ?" s'écrie le roi. "Quelle ést sa patrie ? quel est ce vêtement ?» ${ }^{42}$ La suite de la scène parodique chez Aristophane qui nous fait connaître ces vers suggère que

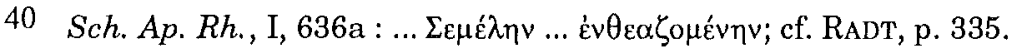

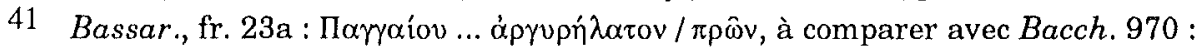

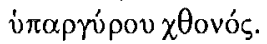

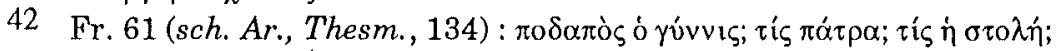


le Dionysos d'Eschyle tenait une lyre et portait la fine crocote orientale ${ }^{43}$. Lycurgue se moque aussi de ses "longues jambes» 44 . Le chitôn lydien est encore évoqué dans un autre fragment, sans doute porté sur la bassara "qui lui tombe jusqu'aux pieds» ${ }^{45}$. Avant qu'il n'entre en scène, le chœur des Édoniens décrit son cortège d'hommes et de femmes. Ceux-ci ont l'air, dit-il, "de pratiquer les saintes orgies de Cotytô» 46 , c'est-à-dire de la déesse thrace proche d'Artémis-Bendis. La description lyrique du chœur continue ainsi : "L'un tient en main une flûte grave, instrument fait au tour, et répand une mélodie modulée par ses doigts, le chant qui éveille la frénésie. Un autre fait vibrer ses cymbales de bronze... Les cordes résonnent. Et imitant la voix du taureau, des histrions ${ }^{47}$ produisent, venant d'on ne sait où, de sourds mugissements, tandis que le tambour, en écho, fait rouler, comme s'il venait de sous terre, le grondement terrifiant du tonnerre».

Strabon, qui cite ces vers, remarque qu'ils s'appliqueraient bien à des Phrygiens mais que ceux-ci étant des «colons» des Thraces, qui identifiaient Dionysos avec «le Lycurgue édonien», il était naturel qu'il y ait une fusion des rites. Ce morceau, outre sa beauté formelle, est d'un vif intérêt pour le rituel. On aura remarqué l'insistance du poète sur les tonalités graves ou sourdes des instruments et des chants, qui créent une atmosphère mystérieuse. Les voix imitant le mugissement des boeufs rappellent le motif du Dionysos-Taureau, tandis que les roulements de tambour imitant l'orage renvoient à Bromios, le dieu grondant. La musique de la bacchanale reparaît encore dans un court fragment non localisé : "Au plus fort retentissent toutes les cymbales» 48 . Dans un autre fragment lyrique, le chœur s'écriait : «il convient d'entonner un dithyrambe mêlé de cris, en union avec le cômos de Dionysos» ${ }^{49}$. Les Xantriai citent encore "les torches de pin enflammées» 50 , celles qui éclairent les rites nocturnes des Bacchantes. Un dernier fragment, d'une pièce incon-

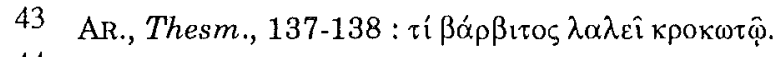

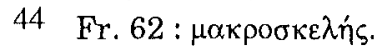

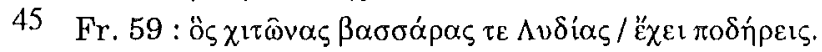

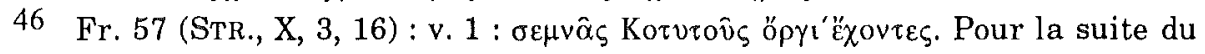
fragment, on comparera la parodos des Bacchantes, 155-165.

$47 \mu \hat{i} \mu \mathrm{ol}$.

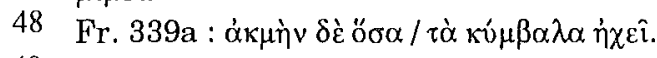

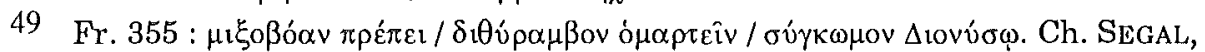
Dionysiac Poetics and Euripides' Bacchae, Princeton, 1982, p. 57, a insisté sur l'importance dans le drame d'Euripide du thème de la "communion" du dieu et de ses fidèles, marqué par la préposition oúv.

50

Fr. 171. 
nue, porte cette invocation à Dionysos : «Père, dieu du vin, toi qui mets les Ménades sous ton joug !» 51 L'épithète cultuelle employée ici, Théoïnos, est extrêmement rare, et rappelle la fête attique, elle aussi très mal connue, des Théoïnia. Peut-être ces vers venaient-ils du Lycurgue, où il semble que se trouvaient comparés les mérites respectifs de la bière et du vin...

De telles citations mettent en évidence l'aspect bienveillant du dieu «très doux» 52 , comme dit Euripide, à l'égard de ses fidèles. Pourtant, le point commun de toutes les pièces dionysiaques d'Eschyle est d'être des "drames de refus», marqués par la résistance des hommes et des femmes, soit à accepter l'instauration de son culte, soit à participer à ce culte déjà établi, avec pour corollaire la persécution du dieu et de ses fidèles. Or la vengeance de Dionysos est presque toujours d'une extrême cruauté : c'est le cas pour Lycurgue, Orphée, les filles de Minyas, Penthée. Les mythes conservent encore d'autres récits parallèles concernant les filles de Prœtos, celles d'Eleuther, le Thrace Boutès, ou même les pirates tyrrhéniens dans l'Hymne homérique 53 .

Rappelons comment se comporte alors le dieu. Au début, avec une passivité et une douceur trompeuses : c'est le cas des Édoniens, comme plus tard dans les Bacchantes. Mais ensuite, il apparaît vite que les mortels n'ont pas de prise sur lui. Il glisse hors des chaînes, il s'échappe des cachots avec ses bacchantes. Insaisissable, il change de forme, avec une prédilection pour certaines incarnations animales : lion, taureau, léopard, serpent ${ }^{54}$. Ainsi, un vers des Bassarides laisse à penser que Dionysos prenait dans la pièce l'apparence d'un taureau furieux qui pointait ses cornes contre un ennemi ${ }^{55}$. Les pouvoirs humains trouvent leur limite devant la puissance bacchique qui pénètre l'univers : dans l'Hymne homérique à Dionysos, à l'appel du dieu "une rumeur immense envahit la forêt» ${ }^{56}$; dans les Bacchantes, ce sont la terre et la montagne qui dansent ${ }^{57}$. Dans les Édoniens, la frénésie gagne le palais royal, qui vacille et tournoie comme une bacchante, ce que le poète exprime dans un vers d'une extrême puissance dans sa concision : "La demeure est possédée du dieu, le toit danse la bacchanale» ${ }^{58}$. De plus, le dieu entoure le

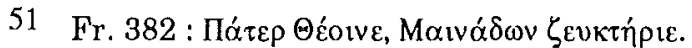

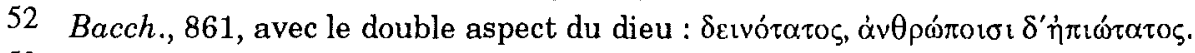

53 Sur ce caractère de cruauté de Dionysos, cf. A. MOREAU, op. cit, p. 216-218.

54 Cf. É. COCHE DE LA FERTÉ, op. cit., p. 222

55 Fr. 23 : кupi $\zeta \varepsilon ı v . ~ S u r$ Dionysos-Taureau, cf. Bacch, 618; 920; 1017; 1159.

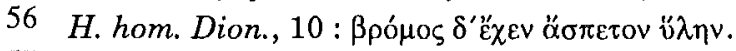

57 Bacch., 726.

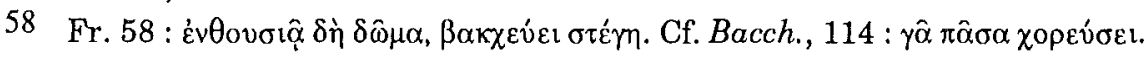


palais de flammes ${ }^{59}$. Ces deux prodiges ont été repris par Euripide, mais peut-être avec moins d'éclat.

On ne sait pas toujours du reste, si ces miracles sont réels ou le fruit d'une hallucination, et ce motif tient une place importante dans les Bacchantes. Dans les Xantriai, d'après les sources indirectes, les filles de Minyas croyaient voir leur maison envahie par des bêtes sauvages, le lierre et les pampres s'enrouler autour de leur métier à tisser d'où s'égouttaient le lait et le miel. Affolées par des visions terrifiantes, elles étaient frappées de mania, la folie homicide conduisant au meurtre, au diasparagmos et à l'omophagie. Lui aussi atteint d'hallucination, Lycurgue empoignait son fils en croyant saisir un sarment de vigne qu'il «taillait» à grands coups de hache ${ }^{60}$. De même l'Agavé des Bacchantes allait déchirer son fils en croyant démembrer un lion...

Mais Dionysos dispose encore d'une "arme secrète» qui se substitue à lui pour détruire ses ennemis, en la personne de Lyssa, la divinité de la rage, de la démence meurtrière. Avec les Érinyes - si présentes également dans le théâtre d'Eschyle - Lyssa devait appartenir à un très ancien fonds religieux, mais Eschyle paraît bien avoir été le premier à la porter sur le théâtre. Il l'avait utilisée dans plusieurs autres pièces ${ }^{61}$, mais elle figurait surtout dans les drames du cycle dionysiaque : nous avons vu que les peintres de vases attiques la montraient aiguillonnant Lycurgue contre son fils et sa femme. Dans les Cardeuses, c'était encore Lyssa qui se lançait avec les Ménades d'Orchomène à la poursuite des fille de Minyas. Mais dans la pièce elle expliquait elle-même qu'elle agissait ainsi parce qu'elle était "possédée par le dieu» et elle décrivait aux bacchantes le processus de l'action divine : «Depuis les pieds, un spasme (sparagmos) va montant jusqu'au sommet de ma tête : l'aiguillon d'une langue, je veux dire le dard d'un scorpion» 62 . Ce n'est sans doute pas un hasard si ce même mot de sparagmos désigne à la fois le spasme qui déchire les chairs et le démembrement des victimes des bacchantes ${ }^{63}$ - Penthée, Orphée ou le fils de la Minyade. Lyssa est encore évoquée dans les Bacchantes, où les

59 NAEVIUS, fr. 24-25 Sutton; pour les Bacchantes, cf. supra, p. 72 et n. 11.

60 Apoll., III, 5, 1.

61 Peut-être dans les Lemniennes, dans Ixion et dans les Perrhaibides (fr. 314b M.) sûrement dans les Toxotides. On rappellera en passant que les Érinyes sont qualifiées de Ménades dans les Euménides (500). Sur Lyssa chez Eschyle, voir J. DUCHEMIN, in REG, 80 (1967), p. 130-139.

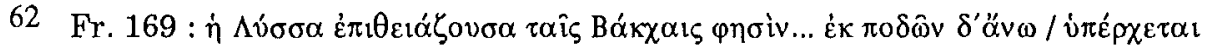

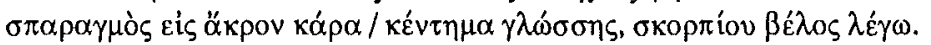

63 Bacch., 735; 739; 1120; 1135. 
Ménades sont appelées les «chiennes de chasse de Lyssa»64, mais ici la figure s'efface derrière le symbole - ou la métaphore. En revanche, dans son Héraclès, Euripide a tiré un grand parti dramatique du personnage eschyléen. Comme l'a montré naguère D.F. Sutton ${ }^{65}$, la seconde moitié de ce drame semble calquée plus directement qu'on ne le pensait sur la scène du meurtre familial de la Lycurgie. On peut estimer que chez Eschyle le recours à cette personnification a découlé de l'impuissance du poète à justifier par le recours à la psychologie humaine le déchaînement de forces si manifestement surnaturelles.

Il arrive enfin que de brefs fragments de ces drames, dans leur dépouillement et leur absence de contexte, nous présentent des affirmations déconcertantes, qui nous rappellent, s'il en était besoin, combien complexe est la personnalité religieuse de Dionysos ${ }^{66}$. Ainsi, par exemple, pour le très énigmatique vers des Bassarides qui paraît bien désigner ce dieu : "L'Apollon au lierre, le devin bacchique» 67. Ainsi encore pour un fragment des Édoniens qui semble lui aussi désigner Bacchos, en le qualifiant de mousomantis ${ }^{68}$, devin-chanteur ou poète, épithète qui conviendrait mieux à Orphée ou à Apollon. De telles invocations débordaient sans doute le cadre dramatique pour exprimer une tradition religieuse comprise et sentie par le public d'Eschyle, mais dont le sens nous échappe en grande partie. En fait, l'historien des idées religieuses en sait de nos jours à la fois plus et moins qu'Eschyle sur les mythes qu'il a mis en scène, et c'est sur ce point que je voudrais conclure.

Dans la mesure où ils nous étaient accessibles, nous avons envisagé l'action tragique et les vers d'Eschyle en quelque sorte au premier degré, sans nous interroger sur les rapports du drame avec l'histoire des mythes

64 Bacch., 977.

65 D.F. SutTon (1975), p. 359-360; voir aussi F. Jovan, Le Prométhée d'Eschyle et l'Héraclès d'Euripide, in REA, 72 (1970), p. 317-331.

66 L'étude de Ch SEGAL (citée n. 49) met l'accent sur les aspects contradictoires de la personnalité de Dionysos.

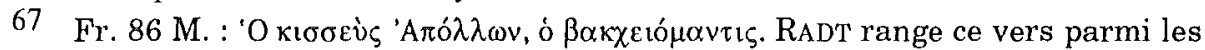
fr. inc. (341). M. WEST, op. cit., p. 70, avoue sa perplexité devant ce vers. Le culte de Dionysos Kisseus, lui, est bien attesté dans plusieurs régions de Grèce : cf. A. PrIVITERA, Dioniso in Omero e nella poesia greca arcaica, Rome, 1970, p. 125-126.

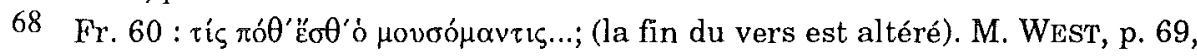
estime que ce sont des paroles de Lycurgue relatives à Orphée. 
et l'existence des rites. Et d'abord l'histoire du culte de Dionysos en Grèce. Dans leur majorité, les chercheurs estiment aujourd'hui que ni l'Orient, ni la Phrygie, ni même la Thrace n'ont été le berceau des cultes dionysiaques, mais que ceux-ci, très anciennement établis en Grèce, ont été au contraire introduits par les Grecs dans ces pays ${ }^{69}$. Cette introduction a été favorisée en Thrace par la présence de divinités et de rites proches de ceux qui leur étaient familiers. Ainsi, comme l'avait pressenti Strabon ${ }^{70}$, Dionysos et Lycurgue ont moins été des adversaires politiques que des rivaux religieux, avant de se fondre en une seule divinité, DionysosLycurgue l'Édonien. Quant au Lycurgue emprisonné dans sa caverne, il est étrangement proche de cet anthropodaimôn évoqué par la Muse à la fin du Rhésos, "caché dans les antres de la terre riche en argent, prophète de Bacchos habitant la roche du Pangée, du dieu vénéré de ceux qui savent» ${ }^{71}$. Autre exemple : si le culte du Soleil est un fait religieux thrace, attesté entre autres dans un fragment de Sophocle, un autre fragment du même poète nous indique qu'Apollon-Hélios était aussi honoré à Athènes, sans doute dans certains milieux pénétrés d'influences orphico-pythagoriciennes $^{72}$, et cela, le poète des Bassarides ne l'ignorait sûrement pas. Mais était-il conscient, par exemple, qu'avant d'être une princesse thébaine, Sémélé avait été adorée en Béotie très anciennement comme une divinité chthonienne ? ${ }^{73}$ Sentait-il le caractère d'hiéroi logoi des mythes apologétiques contant la défaite des adversaires du dieu et reflétant sans doute un difficile établissement de certains rites dionysiaques dans les cités grecques $?^{74}$ Mesurait-il tout ce que devaient au rituel des schémas mythiques du type : résistance au dieu, folie, poursuite, meurtre, démembrement et omophagie, que des fêtes comme les Agrionies d'Orchomène et d'ailleurs répétaient à son époque sous une forme atténuée et symbolique ? ${ }^{75}$ Il n'est pas douteux qu'Eschyle, initié à Éleusis,

69 Cf. G.A. Privitera, op. cit.

70 Cf. supra, p. 81 et n. 46.

71 EuR, Rhes., 971-973 : sur ces vers, voir J. DigGLE, in SIFC, 3e sér., 5 (1987), p. 167-172; voir aussi HDT., VII, 111 (sanctuaire oraculaire de Dionysos chez les Thraces).

72 Respectivement fr. 582 Radt (Térée) et fr. inc. 752; et $O . R ., 660-661$. Sur ce dernier point, voir P. BoYancé, in Mél. Carcopino, Paris, 1966, p. 149-170.

73 E. COCHE DE LA FERTÉ, op. cit., p. 215 et 237; déjà H. JEANMAIRE, op. cit., p. 343-345.

74 Voir les réflexions de R. SEAFond, Dionysiac Drama and the Dionysiac Mysteries, in CQ, 31 (1981), p. 252-275.

75 Sur les Agrionies, voir M. DARAKI, Aspects du sacrifice dionysiaque, in $R H R$, 31 (1981), p. 138-142. 
connaissait d'autres pratiques secrètes et d'autres mystères, et il devait respecter, peut-être même pratiquer certains d'entre eux, sans les analyser. Entre ces deux modes de connaissance, il serait vain d'établir une hiérarchie.

Université de Paris X-Nanterre

François JOUAN 200, av. de la République

F - 92001 NANTERRE Cedex 